\title{
Location Optimization for Square Array Antennas Using Differential Evolution Algorithm
}

\author{
Erkan Afacan \\ Department of Electrical \& Electronics Engineering, Faculty of Engineering, Gazi University, Maltepe, 06570 Ankara, Turkey \\ Correspondence should be addressed to Erkan Afacan; e.afacan@gazi.edu.tr
}

Received 28 March 2016; Accepted 24 April 2016

Academic Editor: Sotirios K. Goudos

Copyright (C) 2016 Erkan Afacan. This is an open access article distributed under the Creative Commons Attribution License, which permits unrestricted use, distribution, and reproduction in any medium, provided the original work is properly cited.

In recent works thinned arrays giving minimum peak sidelobe levels for planar square antenna arrays are obtained using Hadamard difference sets. In the current work thinned array configurations giving lower peak sidelobe levels than those given in the literature are obtained for square arrays of $6 \times 6,8 \times 8,12 \times 12$, and $16 \times 16$ elements. Differential evolution algorithm is used in the determination of the antenna locations.

\section{Introduction}

Thinned antenna arrays offer advantages of cost, weight, and power consumption. If the array elements are located periodically then it is difficult to control sidelobe level (SLL) effectively. One way of overcoming this problem is to place array elements aperiodically. So, a planar antenna array can be placed on a two-dimensional grid with equal spacing. For thinning, some of the antenna elements from the array are removed or turned off, and the remaining active elements are fed with equal amplitude currents.

Since the synthesis problem for planar thinned arrays is complex and cannot be solved analytically, several global optimization algorithms are proposed in the literature such as simulated annealing [1], ant colony optimization [2], invasive weed optimization [3], genetic algorithm [4-7], particle swarm optimization [8,9], differential evolution [10], and teaching learning based optimization algorithm [11].

In [1], simulated annealing is applied to thinning and weighting of planar arrays with element numbers as large as $64 \times 64$. Ant colony optimization is applied to a $20 \times 10$ elements' planar array in [2], and the SLLs in $\phi=0^{\circ}$ and $\phi=90^{\circ}$ planes are sought to be minimized. Invasive weed optimization which is inspired from the phenomenon of colonization of invasive weeds in nature is applied to a $20 \times 10$ elements' planar array in [3].

In [4] a $20 \times 10$ elements' planar antenna array is optimized by using genetic algorithm and a thinned array filled 54\% with 108 elements is obtained. Since the problem geometry is symmetric optimization is realized for a $10 \times 5$ elements' antenna array in fact. A multiple agent optimization technique based on genetic algorithm is presented in [5]. Authors have reduced the multiobjective problem into a classical single objective one, so it has been possible to specify multiple constraints within the method. The proposed method is applied to $12 \times 12$ and $20 \times 10$ elements' planar arrays. In [6] the same problem given in [4] is studied with modified real genetic algorithm and a thinned array filled $50 \%$ with 100 elements is obtained. A genetic algorithm based on orthogonal design is utilized to optimize the peak sidelobe level (PSLL) of a $20 \times 10$ elements' planar array in [7].

In [8] binary and real particle swarm optimizations are used to synthesize a $20 \times 10$ elements' planar array with two different cost functions. In [9] a feedback based binary particle swarm optimization is proposed. The algorithm is applied to $6 \times 6,12 \times 12$, and $24 \times 24$ elements' square arrays. The cost function is taken as a linear combination of PSLL and thinning percentage.

Differential evolution (DE) algorithm is a widely used algorithm in antenna array synthesis problems for linear [1219], circular [20-22], planar [10, 23-25], and conformal arrays [26]. DE is also applied to Yagi-Uda antennas in $[27,28]$.

In [12], DE algorithm is applied to the synthesis of low sidelobe linear antenna arrays. Authors have shown that DE converges faster than real-coded genetic algorithm. DE algorithm is applied to the synthesis of uniform amplitude, 
unequally spaced linear arrays in [13]. Position-only, phaseonly, and position-phase syntheses are considered and it is observed that position-phase synthesis gives better results than two others. The effect of element spacing and element number on SLL is investigated in detail. In [14], DE algorithm is used for linear array pattern synthesis with prescribed nulls at the direction of interferences while SLLs are kept below desired levels. Only the array element excitation amplitudes are controlled. A pattern synthesis method based on DE is proposed for uniform amplitude thinned linear phased arrays in [15]. The locations and phase excitations of the thinned array elements are optimized. Reduction of grating lobes and minimization of SLLs are aimed at while keeping the main beam pointed to the desired direction. In [16], time modulation is used for the design of unequally spaced linear arrays. DE algorithm is adopted to optimize the positions and switch-on times of elements in the array, and SLLs and sideband levels are tried to be suppressed with beamwidth constraint. A dynamic DE algorithm with the best of random differential mutation is used in the synthesis of unequally spaced linear arrays in [17]. Position-only and position-phase syntheses are considered and lowest PSLLs are investigated. In [18], DE with strategy adaptation is applied to the sparse linear array synthesis. The goal of the optimization is taken as to suppress SLL by finding the optimum element positions and to set main lobe to a desired beamwidth. In [19], composite DE with eigenvector base crossover is applied to shaped beam pattern synthesis problem for linear arrays and for a conformal cylindrical array.

In [20], a numerical approach based on DE algorithm is proposed for the pattern synthesis of concentric ring array antennas with the constrained first null beamwidth. It is shown that the method gives lower PSLLs with the optimization of ring spacing and element number in each ring. A modified form of DE called DE with global and local neighborhoods is used for designing a thinned concentric planar circular antenna array in [21]. Reducing SLL and increasing the number of switched-off elements are aimed at. It is shown that any desired SLL value can be obtained while maintaining the number of switched-off elements at a reasonable value. In [22], a synthesis method for shaping beam pattern of a concentric ring array antenna using DE and genetic algorithm is proposed. Authors have shown that both methods are capable of generating the desired shaped beams.

In [10], a Boolean differential evolution algorithm based on Boolean algebra is proposed. The method has only one control parameter which is the crossover rate. Authors have applied the method to $6 \times 6$ and $10 \times 10$ elements' square arrays, and they have sought the minimum PSLL without the constraint of thinning percentage. The method is also applied to a $20 \times 10$ elements' planar array with a different cost function which is the sum of the maximum SLLs in $\phi=0^{\circ}$ and $\phi=90^{\circ}$ planes. In [23], DE algorithm and a binary-coded genetic algorithm are jointly applied to the minimization of the SLL in a $31 \times 31$ square planar array. It is shown that the SLL of the planar array can be decreased without a large change in directivity. An FFT-based synthesis method for generating dual radiation pattern from a rectangular planar array is presented in [24]. Array elements phases are modified while amplitudes are commonly shared. The FFT method is incorporated with self-adaptive DE algorithm. Different beam pairs having different PSLLs are investigated. The results obtained from self-adaptive DE, DE, and particle swarm optimization are compared with each other. In [25], fractal-based planar antenna array optimization using DE is considered. Sierpinski carpet fractal array concept is utilized and PSLL reduction and element number reduction by finding optimum element positions and amplitudes are aimed at.

In [26], a synthesis method based on DE algorithm is presented for conformal antenna arrays in the presence of a platform. Element phases are determined by the scan angle and array geometry, and element amplitudes are optimized via $\mathrm{DE}$ so as to reduce SLLs to desired levels. In [27], DE is applied to the optimization of the geometric parameters of Yagi-Uda antennas. A hybrid DE algorithm is proposed which incorporates simplified quadratic interpolation in [28]. The method is applied to Yagi-Uda antennas and wideband patch antenna design.

The modified teaching learning based optimization algorithm proposed in [11] is applied to $12 \times 12$ square array and $20 \times 10$ elements' planar array.

Difference sets [29-32] and hybrid methods [33-35] are also introduced for the optimization of planar arrays. In [32], the sidelobe control problem is directly tried to be solved by applying the properties of difference sets. In [29-31], Hadamard difference sets are used to obtain thinned arrays giving minimum SLLs for planar square antenna arrays.

A hybrid approach which combines genetic algorithm and cyclic difference sets is proposed in [34]. Hadamard difference sets based method given in [29] is combined with a binary particle swarm optimization in [33]. The proposed hybrid approach is applied to $6 \times 6,12 \times 12$, and $24 \times 24$ square arrays. The authors have expressed the cost function as a weighted sum of the PSLL and thinning percentage. In [35], McFarland difference sets and a binary genetic algorithm are used for the synthesis of thinned rectangular arrays.

In [36] thinned array configurations giving lower PSLLs than given in [29] were obtained for square arrays of $6 \times 6$ and $8 \times 8$ elements.

In the current work thinned array configurations giving lower PSLLs than given in [29-31] are obtained for square arrays of $6 \times 6,8 \times 8,12 \times 12$, and $16 \times 16$ elements. For the given square arrays, under the constraints of given number of array elements, the optimum thinned array configurations are tried to be found. The obtained PSLLs are also found to be lower than given in [36] for square arrays of $6 \times 6$ and $8 \times 8$ elements. Differential evolution algorithm is used in the determination of the antenna locations.

\section{The Geometry of the Problem}

The planar antenna array under investigation is shown in Figure 1 . The array factor for this planar antenna array can be written as

$$
A F(\theta, \phi)=\sum_{n=1}^{N} \alpha_{n} e^{j 2 \pi x_{n} \sin \theta \cos \phi} e^{j 2 \pi y_{n} \sin \theta \sin \phi} .
$$




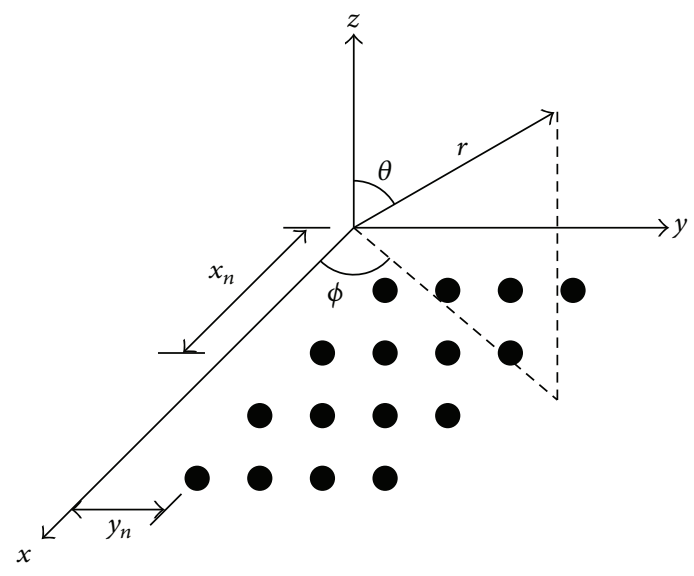

FIGURE 1: Planar antenna array.

Here, $\alpha_{n}$ is the amplitude of the $n$th array element current and it takes the values of 1 or 0 . A value of 1 for $\alpha_{n}$ means that the antenna element is turned on or active, and a value of 0 for $\alpha_{n}$ means that this antenna element is turned off or passive. $N$ is the total number of antenna elements, and $x_{n}$ and $y_{n}$ denote the $x$ and $y$ coordinates of the $n$th element in wavelengths, respectively. The distance between grid points is assumed to be $\lambda / 2$, where $\lambda$ is the wavelength. The array factor given in (1) can be also expressed as a two-dimensional sum, but onedimensional indexing is found to be more efficient from the programming point of view.

The PSLL for the mentioned planar array can be written as

$$
P_{\mathrm{SLL}}=\max _{(\theta, \phi) \in S}\left|\frac{A F(\theta, \phi)}{F F_{\max }}\right|,
$$

where $S$ is the sidelobe region and $F F_{\max }$ is the peak of the main beam. To suppress the PSLL in all planes the cost function to be minimized may be chosen as

$$
f=P_{\mathrm{SLL}}
$$

In this study, the minimization of the PSLLs is aimed at without any other constraint.

\section{The Solution of the Problem with Differential Evolution Algorithm}

In the solution of the problem, differential evolution algorithm is used. DE is a reliable and versatile, population based optimizer [37, 39, 40] and has been widely used in the solution of electromagnetic and antenna problems as mentioned above [10, 12-28, 38, 41, 42].

Using a notation similar to that given in [39], the current population $P_{\mathbf{x}}$ consists of the acceptable vectors $\mathbf{x}_{i, g}$ :

$$
\begin{aligned}
P_{\mathbf{x}, \mathfrak{g}} & =\left(\mathbf{x}_{i, g}\right), \quad i=0, \ldots, N p-1, g=0, \ldots, g_{\max }, \\
\mathbf{x}_{i, g} & =\left(x_{i, g}\right)_{j}, \quad j=0, \ldots, D-1,
\end{aligned}
$$

where $N p$ is the number of the vectors in the population, $D$ is the number of the parameters, $i$ is the population index which runs from 0 to $N p-1, g$ is the generation index running from 0 to $g_{\max }$, and $j$ is the parameter index which runs from 0 to $D-1$.

After initialization, randomly chosen vectors are mutated and intermediate population $P_{\mathbf{v}, g}$ is produced:

$$
\begin{aligned}
& P_{\mathbf{v}, g}=\left(\mathbf{v}_{i, g}\right), \quad i=0, \ldots, N p-1, g=0, \ldots, g_{\max }, \\
& \mathbf{v}_{i, g}=\left(v_{i, g}\right)_{j}, \quad j=0, \ldots, D-1,
\end{aligned}
$$

where $\mathbf{v}_{i, g}$ are mutant vectors. After the recombination of each vector in the current population with a mutant vector, a trial population $P_{\mathbf{u}, g}$ is obtained:

$$
\begin{aligned}
& P_{\mathbf{u}, g}=\left(\mathbf{u}_{i, g}\right), \quad i=0, \ldots, N p-1, g=0, \ldots, g_{\max }, \\
& \mathbf{u}_{i, g}=\left(u_{i, g}\right)_{j}, \quad j=0, \ldots, D-1 .
\end{aligned}
$$

The population is randomly initialized at the beginning (i.e., $g=0$ ). During or before the initialization upper and lower bounds can be specified for the parameters. The initialized population is mutated and recombined to produce the intermediate population. The mutation operator for DE is given as

$$
\mathbf{v}_{i, g}=\mathbf{x}_{r 0, g}+F\left(\mathbf{x}_{r 1, g}-\mathbf{x}_{r 2, g}\right) \text {, }
$$

where $\mathbf{x}_{r 0, g}, \mathbf{x}_{r 1, g}$, and $\mathbf{x}_{r 2, g}$ are three different and randomly chosen vectors and $F$ is the scale factor.

The crossover operation of DE can be shown as

$$
\mathbf{u}_{i, g}=\left(u_{i, g}\right)_{j}= \begin{cases}\left(v_{i, g}\right)_{j}, & \text { if } \gamma \leq \mathrm{Cr}, \\ \left(x_{i, g}\right)_{j}, & \text { otherwise }\end{cases}
$$

where $\mathrm{Cr}$ is the crossover probability $(\mathrm{Cr} \in[0,1])$ and $\gamma \in$ $[0,1]$ is the outcome of a uniform random number generator. In $\mathrm{DE}$ each vector is crossed with a mutant vector.

After crossover, the selection process is realized as follows:

$$
\mathbf{x}_{i, g+1}= \begin{cases}\mathbf{u}_{i, g}, & \text { if } f\left(\mathbf{u}_{i, g}\right) \leq f\left(\mathbf{x}_{i, g}\right), \\ \mathbf{x}_{i, g}, & \text { otherwise }\end{cases}
$$

where $f$ is the cost function value.

The control parameters for DE are $N p, F$, and Cr. In [37], the following suggestion is given: $N p$ should be between $5 D$ and $10 D$, and $F=0.5$ is a good choice for beginning, but if the population converges prematurely then $F$ and/or $N p$ should be increased. $\mathrm{Cr}=0.9$ or $\mathrm{Cr}=1.0$ is advised as an initial choice. In [38], it is stated that a good initial choice is $F=0.6$. In the literature several values for these control parameters are used. After a moderate survey, Table 1 is obtained.

The cost function is chosen as given in (3) and the antenna configurations are searched which minimize the PSLLs.

In the problem under consideration it is necessary to calculate sidelobe levels in 3 dimensions for $0 \leq \phi<360^{\circ}$ 
TABLE 1: DE control parameters as used in the literature.

\begin{tabular}{lccc}
\hline Reference & $\mathrm{Np}$ & $F$ & $\mathrm{Cr}$ \\
\hline$[37]$ & {$[5 D, 10 D]$} & 0.5 & 0.9 or 1.0 \\
\hline$[12,14,16]$ & $5 D$ & 0.6 & 0.9 \\
\hline$[15]$ & {$[4 D, 5 D]$} & {$[0.5,0.7]$} & {$[0.7,0.9]$} \\
\hline$[17]$ & 60 & 0.5 & 0.8 \\
\hline$[18]$ & 50 & 0.5 & 0.9 \\
\hline$[19]$ & & 0.5 & 0.9 \\
\hline$[20]$ & 100 & 0.5 & 0.9 \\
\hline$[22]$ & 50 & 0.8 & 0.2 \\
\hline$[23]$ & 100 & 0.5 & \\
\hline$[24]$ & 50 & 0.8 & 0.7 \\
\hline$[25]$ & $20 D$ & 0.5 & 0.8 \\
\hline$[27]$ & 70 & 0.8 & 0.8 \\
\hline$[28]$ & & 0.5 & 0.9 \\
\hline$[38]$ & {$[3 D, 8 D]$} & {$[0.4,1.0]$} & {$[0.3,0.9]$} \\
\hline
\end{tabular}

and $0 \leq \theta \leq 90^{\circ}$. This process brings a heavy computational burden. To speed up the calculations, the array factor is defined as a three-dimensional global variable and is calculated only once before the beginning of the iterations for generations and populations. So, a trade-off is maintained between memory and speed.

\section{Numerical Results and Comparison with the Literature}

At first, PSLLs are tried to be minimized for $6 \times 6$ and $8 \times 8$ square arrays so as to be able to compare the results with $[29,30,33,36]$. For the $6 \times 6$ square array the total number of active antenna elements is taken as $N=15$ and $N=21$. In a similar manner, for the $8 \times 8$ square array the total number of active antenna elements is taken as $N=28$ and $N=36$. The parameters of the differential evolution algorithm are taken as follows: scale factor $F=0.6$, crossover probability $\mathrm{Cr}=0.9$, maximum number of generations $g_{\max }=200$, and population size $N p=5 N$. The selection of these parameter values is based on the literature $[12,14,16]$ and on the experience of the author. After numerous experiments, the strategy "DE/rand/1/bin" has been found to be the most effective strategy. For each case at least 250 trials are run and the best result is chosen. The array configurations obtained at the end of the simulations are shown in Figures 2-5. PSLLs obtained in this work are compared with the values given in $[29,30,33,36]$ in Table $2.6 \times 6$ planar antenna array is also investigated in [10] without the constraint of thinning percentage and a thinned array with 30 elements having a PSLL of $-16.64 \mathrm{~dB}$ is obtained. Since the element numbers used in this work and in [10] are different, it is not meaningful to compare the results.

Secondly, PSLLs are tried to be minimized for $12 \times 12$ and $16 \times 16$ square arrays. The total number of active antenna elements is taken as $N=66$ and $N=78$ for the $12 \times 12$ square array and as $N=120$ and $N=136$ for the $16 \times 16$ square array. The parameters of the differential evolution algorithm

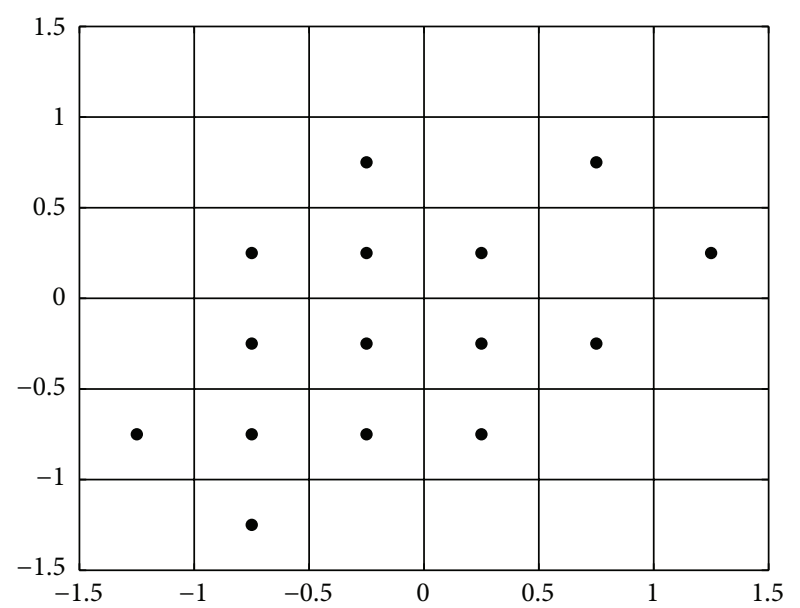

Figure 2: Array configuration for 15 elements.

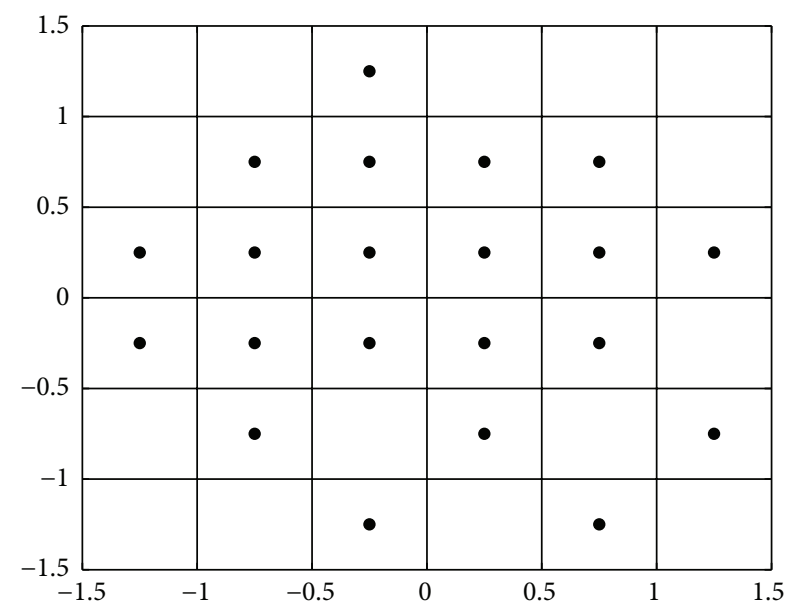

FIGURE 3: Array configuration for 21 elements.

TABLE 2: Peak sidelobe levels for square arrays of $6 \times 6$ and $8 \times 8$.

\begin{tabular}{lcccc}
\hline$N$ (antenna number) & 15 & 21 & 28 & 36 \\
\hline PSLL (dB) [29] & -10.18 & -12.55 & -12.46 & -13.71 \\
\hline PSLL (dB) [30] & & & -12.59 & -13.86 \\
\hline PSLL (dB) [33] & -13.06 & & & \\
\hline PSLL (dB) [36] & -13.27 & -16.11 & -13.56 & -15.76 \\
\hline PSLL (dB) [this work] & -14.40 & -16.28 & -17.64 & -18.35 \\
\hline
\end{tabular}

TABLE 3: Peak sidelobe levels for square arrays of $12 \times 12$ and $16 \times 16$.

\begin{tabular}{lcccc}
\hline$N$ (antenna number) & 66 & 78 & 120 & 136 \\
\hline PSLL (dB) [29] & -14.91 & -15.47 & -15.71 & -15.17 \\
\hline PSLL (dB) [31] & & & -16.59 & -16.60 \\
\hline PSLL (dB) [this work] & -19.49 & -20.55 & -20.08 & -21.08 \\
\hline
\end{tabular}

are taken as given above. The array configurations obtained as a result of the simulations are shown in Figures 6-9. PSLLs are given in Table 3. In Table 3, the results given in $[29,31]$ are also presented for the purpose of comparison. 


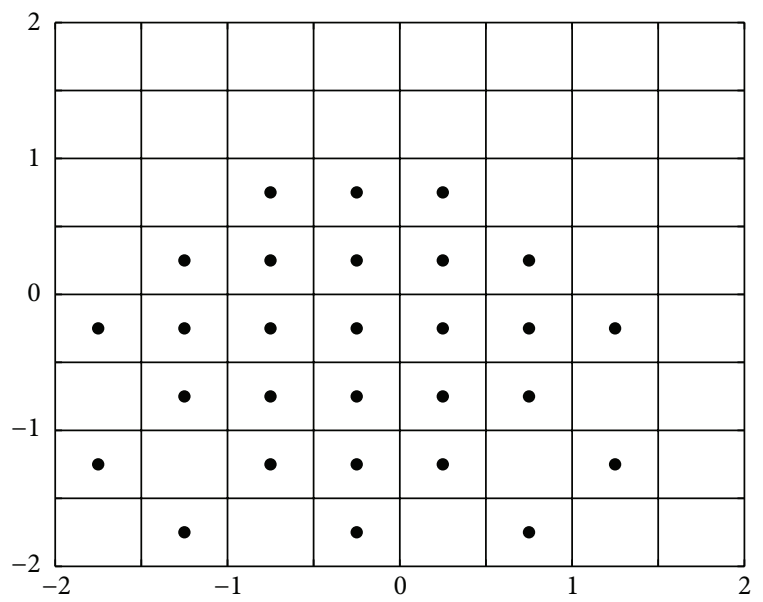

FIGURE 4: Array configuration for 28 elements.

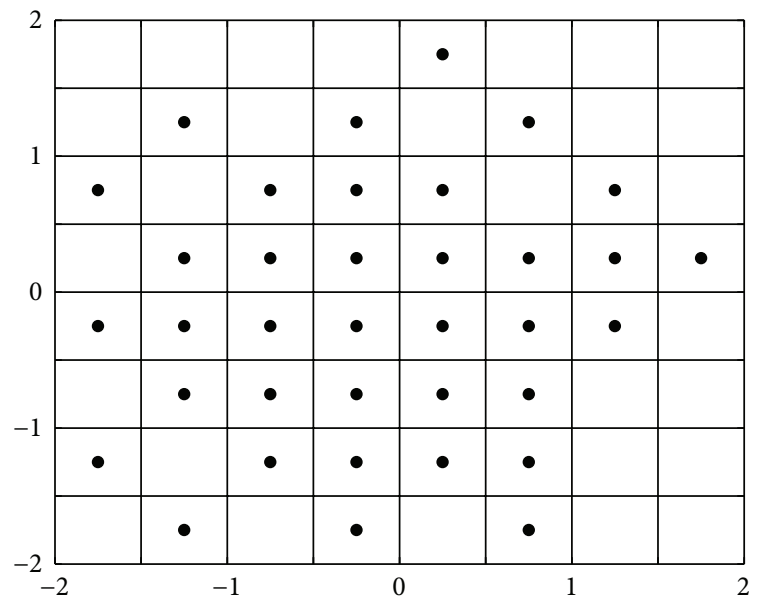

Figure 5: Array configuration for 36 elements.

In [5], for $12 \times 12$ planar array, a configuration is obtained consisting of 64 active elements with a PSLL of $-24.56 \mathrm{~dB}$. Since in the mentioned work, weighting of the array element amplitudes is also allowed, it is not unexpected that the PSLL for 64 active elements is less than the PSLL for 66 active elements without amplitude weighting given in this work.

From Tables 2 and 3, it is seen that there is a significant decrease for PSLLs. Three- dimensional radiation pattern for the 136 elements' antenna array configuration given in Figure 9 is shown in Figure 10.

For further reduction of the sidelobe level, time modulation can be applied as a second step. For example, in [43] time modulation is applied to a 15-element antenna array and it is found that the peak sidelobe can be lowered to $-16.00 \mathrm{~dB}$.

\section{Conclusion}

In this work it is shown that it is possible to obtain planar square antenna array configurations having lower peak sidelobe levels than given in the literature by using differential evolution algorithm. The methods based on difference sets are rigorous but limited to certain geometries, whereas global

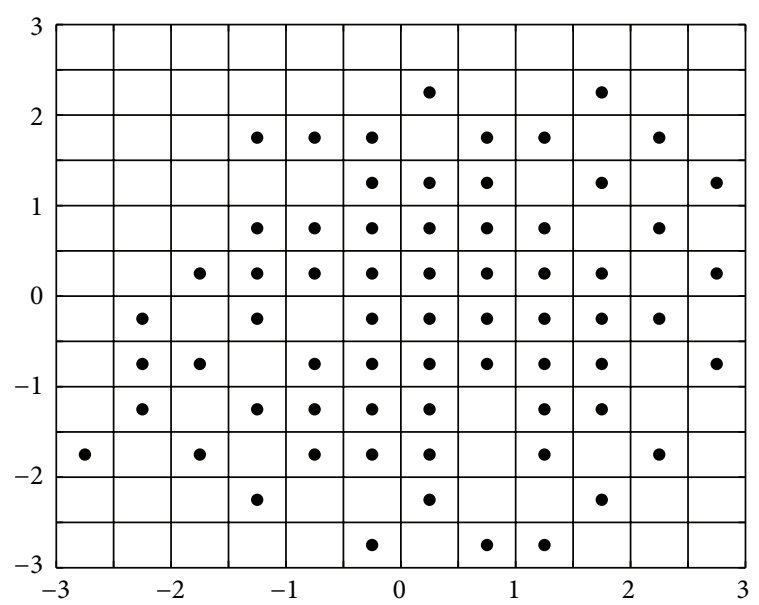

FIgURE 6: Array configuration for 66 elements.

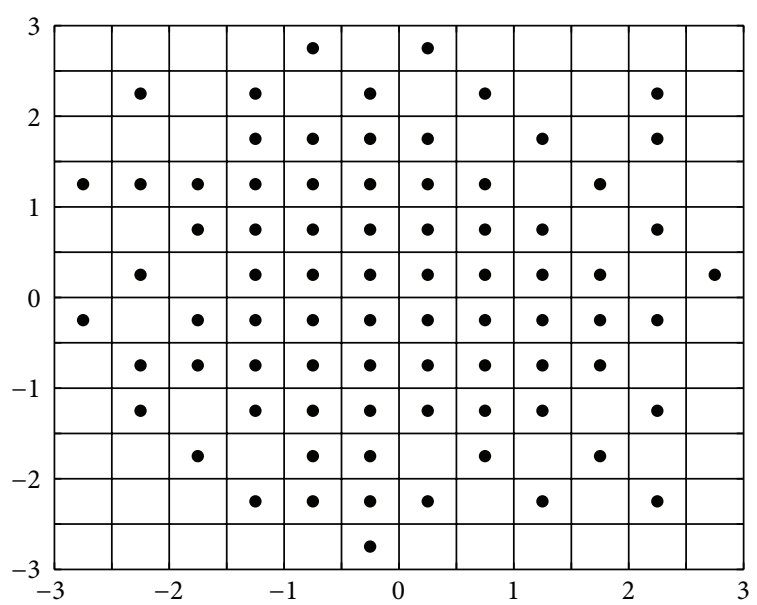

Figure 7: Array configuration for 78 elements.

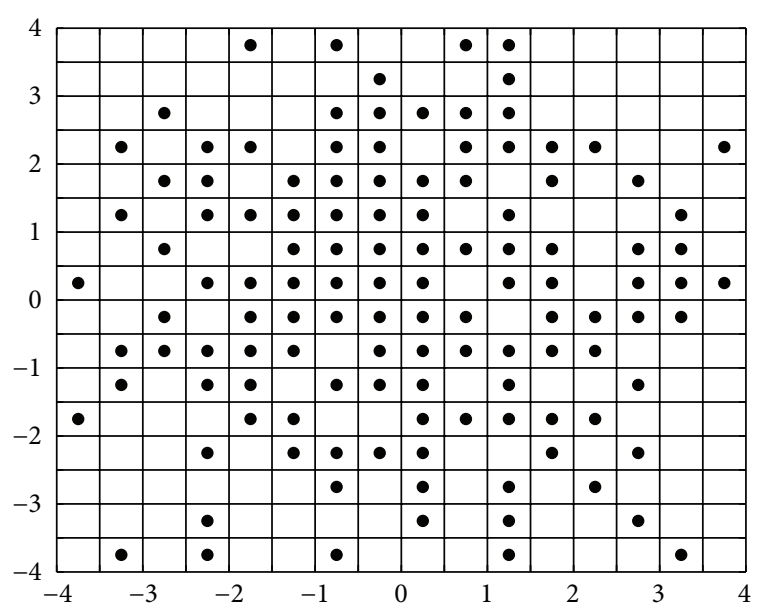

FIGURE 8: Array configuration for 120 elements.

optimization algorithms such as differential evolution can be applied for arbitrary array configurations. 


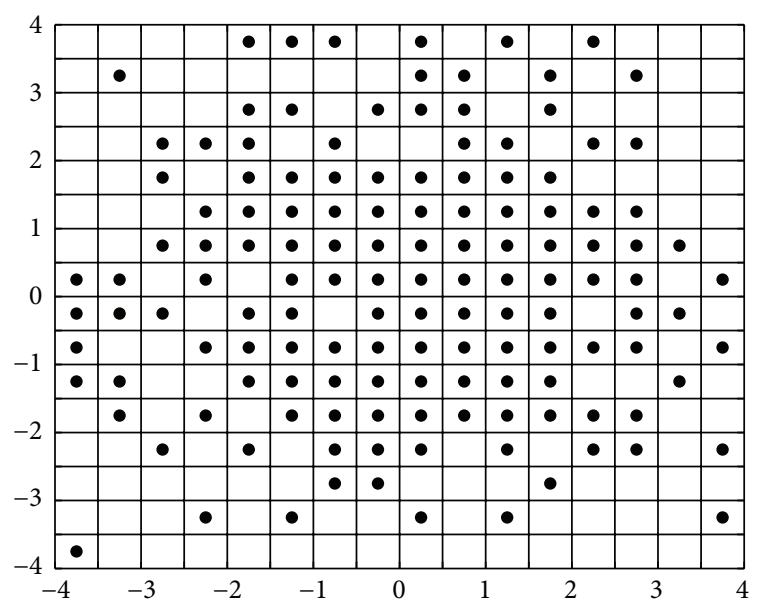

FIgURE 9: Array configuration for 136 elements.

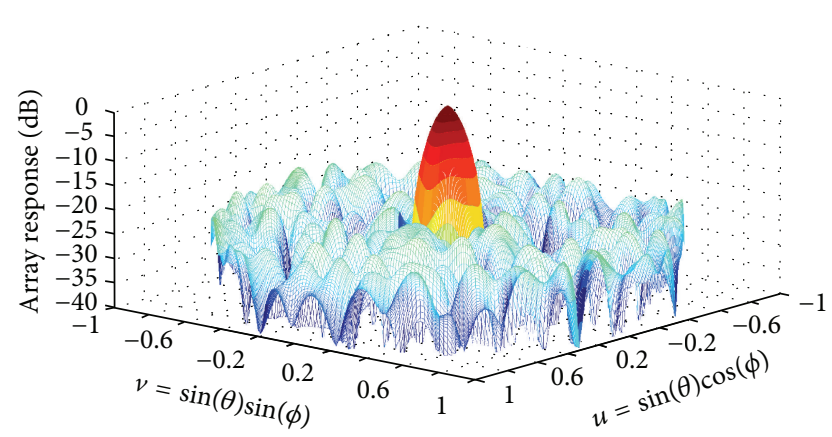

FIGURE 10: Three-dimensional radiation pattern for 136 elements' antenna array given in Figure 9.

\section{Competing Interests}

The author declares that there is no conflict of interests regarding the publication of this paper.

\section{References}

[1] A. Trucco, "Thinning and weighting of large planar arrays by simulated annealing," IEEE Transactions on Ultrasonics, Ferroelectrics, and Frequency Control, vol. 46, no. 2, pp. 347-355, 1999.

[2] O. Quevedo-Teruel and E. Rajo-Iglesias, "Ant colony optimization in thinned array synthesis with minimum sidelobe level," IEEE Antennas and Wireless Propagation Letters, vol. 5, pp. 349$352,2006$.

[3] S. Karimkashi and A. A. Kishk, "Invasive weed optimization and its features in electromagnetics," IEEE Transactions on Antennas and Propagation, vol. 58, no. 4, pp. 1269-1278, 2010.

[4] R. L. Haupt, “Thinned arrays using genetic algorithms," IEEE Transactions on Antennas and Propagation, vol. 42, no. 7, pp. 993-999, 1994.

[5] M. Donelli, S. Caorsi, F. de Natale, D. Franceschini, and A. Massa, "A versatile enhanced genetic algorithm for planar array design," Journal of Electromagnetic Waves and Applications, vol. 18, no. 11, pp. 1533-1548, 2004.

[6] K. Chen, X. Yun, Z. He, and C. Han, "Synthesis of sparse planar arrays using modified real genetic algorithm," IEEE
Transactions on Antennas and Propagation, vol. 55, no. 4, pp. 1067-1073, 2007.

[7] L. Zhang, Y.-C. Jiao, B. Chen, and H. Li, "Orthogonal genetic algorithm for planar thinned array designs," International Journal of Antennas and Propagation, vol. 2012, Article ID 319037, 7 pages, 2012.

[8] Y. Li, F. Yang, J. Ouyang, Z. Nie, and H. Zhou, "Synthesis of nonuniform array antennas using particle swarm optimization," Electromagnetics, vol. 30, no. 3, pp. 237-245, 2010.

[9] J. Wang, B. Yang, S. H. Wu, and J. S. Chen, "A novel binary particle swarm optimization with feedback for synthesizing thinned planar arrays," Journal of Electromagnetic Waves and Applications, vol. 25, no. 14-15, pp. 1985-1998, 2011.

[10] L. Zhang, Y.-C. Jiao, Z.-B. Weng, and F.-S. Zhang, "Design of planar thinned arrays using a Boolean differential evolution algorithm," IET Microwaves, Antennas and Propagation, vol. 4, no. 12, pp. 2172-2178, 2010.

[11] X. Chen, Z. Luo, X. He, and L. Zhu, "Thinning and weighting of planar arrays by modified teaching-learning-based optimization algorithm," Journal of Electromagnetic Waves and Applications, vol. 28, no. 15, pp. 1924-1934, 2014.

[12] S. Yang, A. Qing, and Y. B. Gan, "Synthesis of low sidelobe antenna arrays using the differential evolution algorithm," in Proceedings of the IEEE Antennas and Propagation Society International Symposium, vol. 1, pp. 780-783, Columbus, Ohio, USA, June 2003.

[13] D. G. Kurup, M. Himdi, and A. Rydberg, "Synthesis of uniform amplitude unequally spaced antenna arrays using the differential evolution algorithm," IEEE Transactions on Antennas and Propagation, vol. 51, no. 9, pp. 2210-2217, 2003.

[14] S. Yang, Y. B. Gan, and A. Qing, "Antenna-array pattern nulling using a differential evolution algorithm," International Journal of $R F$ and Microwave Computer-Aided Engineering, vol. 14, no. 1, pp. 57-63, 2004.

[15] Y. Chen, S. Yang, and Z. Nie, "Synthesis of uniform amplitude thinned linear phased arrays using the differential evolution algorithm," Electromagnetics, vol. 27, no. 5, pp. 287-297, 2007.

[16] G. Li, S. Yang, M. Huang, and Z. Nie, "Sidelobe suppression in time modulated linear arrays with unequal element spacing," Journal of Electromagnetic Waves and Applications, vol. 24, no. 5-6, pp. 775-783, 2010.

[17] C. Lin, A. Qing, and Q. Feng, "Synthesis of unequally spaced antenna arrays by using differential evolution," IEEE Transactions on Antennas and Propagation, vol. 58, no. 8, pp. 2553-2561, 2010.

[18] S. K. Goudos, K. Siakavara, T. Samaras, E. E. Vafiadis, and J. N. Sahalos, "Sparse linear array synthesis with multiple constraints using differential evolution with strategy adaptation," IEEE Antennas and Wireless Propagation Letters, vol. 10, pp. 670-673, 2011.

[19] S. K. Goudos, "Shaped beam pattern synthesis of antenna arrays using composite differential evolution with eigenvectorbased crossover operator," International Journal of Antennas and Propagation, vol. 2015, Article ID 295012, 10 pages, 2015.

[20] F. Li, Y.-C. Jiao, L.-S. Ren, Y.-Y. Chen, and L. Zhang, "Pattern synthesis of concentric ring array antennas by differential evolution algorithm," Journal of Electromagnetic Waves and Applications, vol. 25, no. 2-3, pp. 421-430, 2011.

[21] P. Ghosh and S. Das, "Synthesis of thinned planar concentric circular antenna arrays-a differential evolutionary approach," Progress in Electromagnetics Research B, vol. 29, pp. 63-82, 2011. 
[22] D. Mandal, A. Chatterjee, T. Mondal, and A. K. Bhattacharjee, "Shaped beam synthesis of concentric ring array antenna using differential evolution algorithm," AEU-International Journal of Electronics and Communications, vol. 69, no. 7, pp. 1025-1031, 2015.

[23] C. Rocha-Alicano, D. Covarrubias-Rosales, C. Brizuela-Rodriguez, and M. Panduro-Mendoza, "Differential evolution algorithm applied to sidelobe level reduction on a planar array," AEU-International Journal of Electronics and Communications, vol. 61, no. 5, pp. 286-290, 2007.

[24] A. Chatterjee and G. K. Mahanti, "Combination of fast Fourier transform and self-adaptive differential evolution algorithm for synthesis of phase-only reconfigurable rectangular array antenna," Annals of Telecommunications, vol. 69, no. 9, pp. 515527, 2014.

[25] A. Karmakar, R. Ghatak, R. K. Mishra, and D. R. Poddar, "Sierpinski carpet fractal-based planar array optimization based on differential evolution algorithm," Journal of Electromagnetic Waves and Applications, vol. 29, no. 2, pp. 247-260, 2015.

[26] J.-L. Guo and J.-Y. Li, "Pattern synthesis of conformal array antenna in the presence of platform using differential evolution algorithm," IEEE Transactions on Antennas and Propagation, vol. 57, no. 9, pp. 2615-2621, 2009.

[27] J.-Y. Li and J. L. Guo, "Optimization technique using differential evolution for Yagi-Uda antennas," Journal of Electromagnetic Waves and Applications, vol. 23, no. 4, pp. 449-461, 2009.

[28] L. Zhang, Y.-C. Jiao, H. Li, and F.-S. Zhang, "Antenna optimization by hybrid differential evolution," International Journal of RF and Microwave Computer-Aided Engineering, vol. 20, no. 1, pp. 51-55, 2010.

[29] L. E. Kopilovich, "Square array antennas based on Hadamard difference sets," IEEE Transactions on Antennas and Propagation, vol. 56, no. 1, pp. 263-266, 2008.

[30] L. E. Kopilovich, "Array antennas of size $8 \times 8$ based on Hadamard difference sets," Radio Physics and Radio Astronomy, vol. 13, no. 2, pp. 210-215, 2008.

[31] L. E. Kopilovich, "Array antennas of size $16 \times 16$ based on Hadamard difference sets," Radiofizika i Radioastronomia, vol. 18, no. 4, pp. 357-361, 2013 (Russian).

[32] D. G. Leeper, "Isophoric arrays-Massively thinned phased arrays with well-controlled sidelobes," IEEE Transactions on Antennas and Propagation, vol. 47, no. 12, pp. 1825-1835, 1999.

[33] M. Donelli, A. Martini, and A. Massa, "A hybrid approach based on PSO and Hadamard difference sets for the synthesis of square thinned arrays," IEEE Transactions on Antennas and Propagation, vol. 57, no. 8, pp. 2491-2495, 2009.

[34] S. Caorsi, A. Lommi, A. Massa, and M. Pastorino, "Peak sidelobe level reduction with a hybrid approach based on GAs and difference sets," IEEE Transactions on Antennas and Propagation, vol. 52, no. 4, pp. 1116-1121, 2004.

[35] G. Oliveri, F. Caramanica, C. Fontanari, and A. Massa, "Rectangular thinned arrays based on McFarland difference sets," IEEE Transactions on Antennas and Propagation, vol. 59, no. 5, pp. 1546-1552, 2011.

[36] E. Afacan, "Antenna location optimization for a square antenna array using differential evolution algorithm," in Proceedings of the 13th National Congress on Electrical-Electronics-Computer and Biomedical Engineering, pp. 201-203, Ankara, Turkey, December 2009 (Turkish).

[37] R. Storn and K. Price, "Differential evolution-a simple and efficient heuristic for global optimization over continuous spaces," Journal of Global Optimization, vol. 11, no. 4, pp. 341359, 1997.

[38] P. Rocca, G. Oliveri, and A. Massa, "Differential evolution as applied to electromagnetics," IEEE Antennas and Propagation Magazine, vol. 53, no. 1, pp. 38-49, 2011.

[39] K. V. Price, R. M. Storn, and J. A. Lampinen, Differential Evolution: A Practical Approach to Global Optimization, Springer, 2005.

[40] S. Das and P. N. Suganthan, "Differential evolution: a survey of the state-of-the-art," IEEE Transactions on Evolutionary Computation, vol. 15, no. 1, pp. 4-31, 2011.

[41] E. Aksoy and E. Afacan, "Planar antenna pattern nulling using differential evolution algorithm," AEU-International Journal of Electronics and Communications, vol. 63, no. 2, pp. 116-122, 2009.

[42] E. Aksoy and E. Afacan, "Thinned nonuniform amplitude time-modulated linear arrays," IEEE Antennas and Wireless Propagation Letters, vol. 9, pp. 514-517, 2010.

[43] E. Afacan, "Sidelobe level and sideband optimization for thinned planar antenna arrays using time modulation," in Proceedings of the 7th European Conference on Antennas and Propagation (EuCAP '13), pp. 336-338, Gothenburg, Sweden, April 2013. 


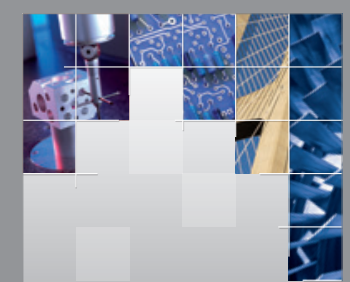

\section{Enfincering}
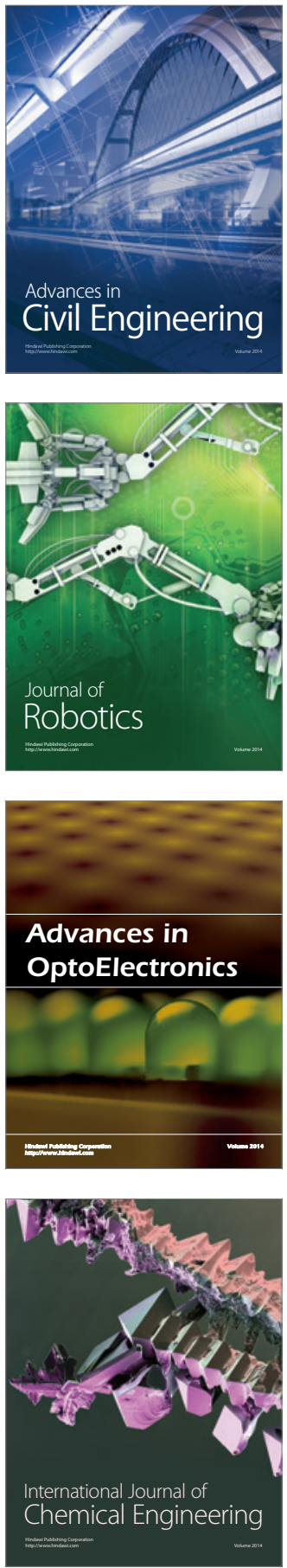

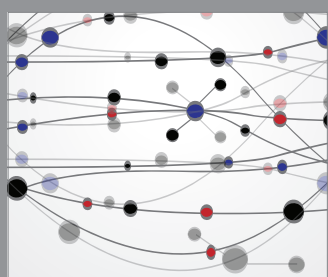

The Scientific World Journal

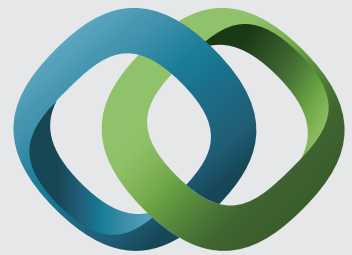

\section{Hindawi}

Submit your manuscripts at

http://www.hindawi.com
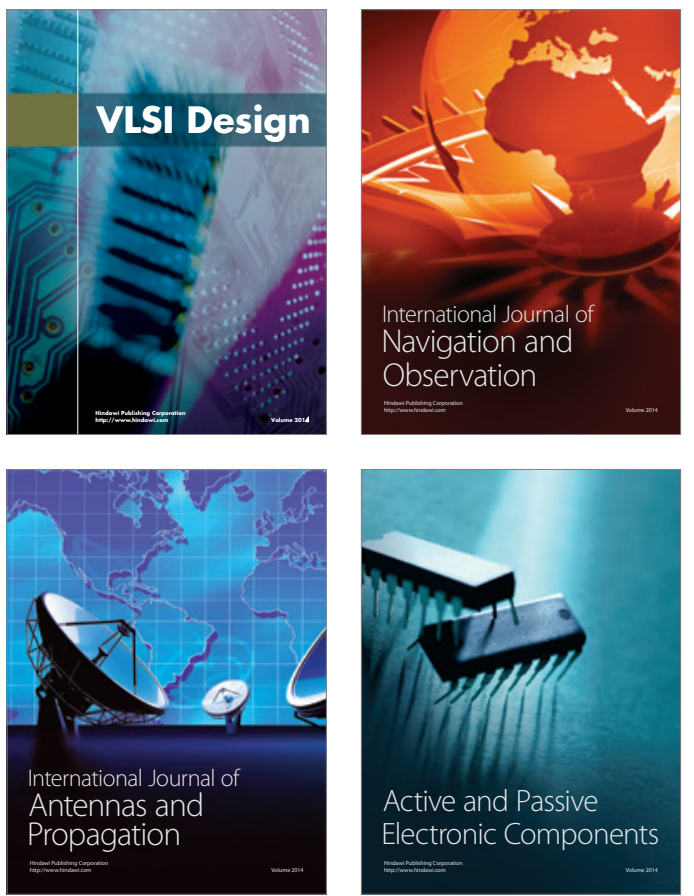
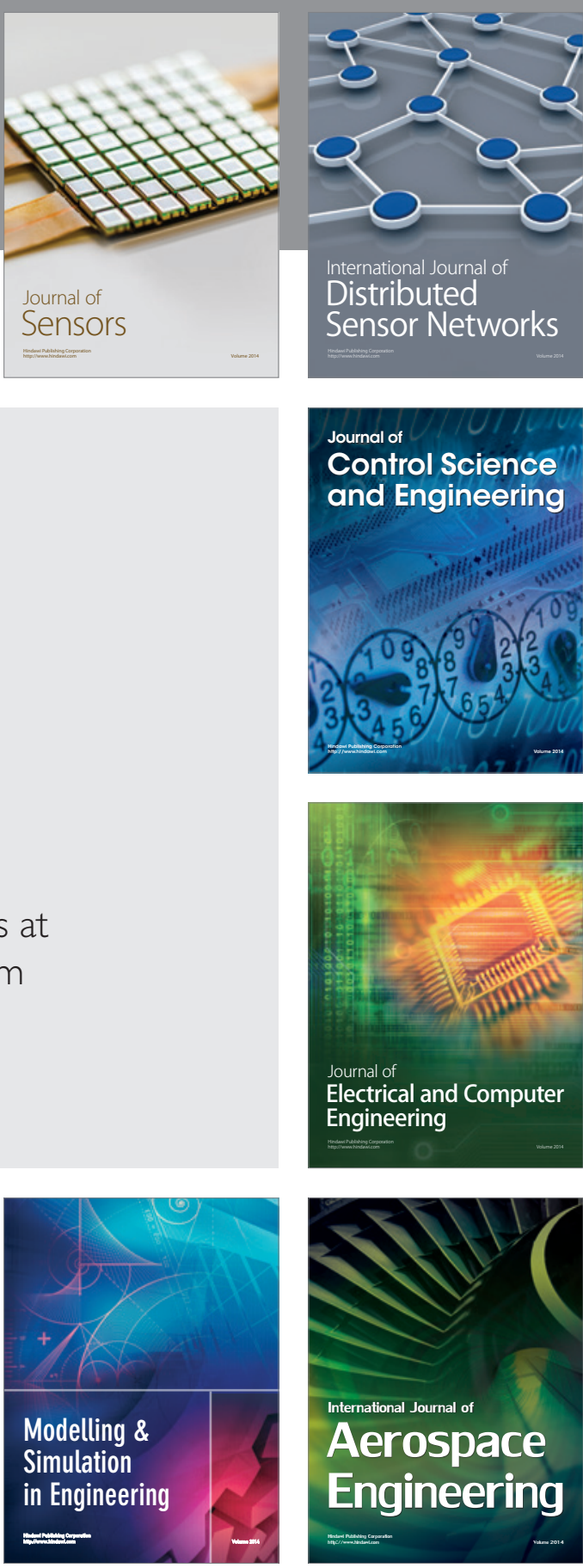

International Journal of

Distributed

Sensor Networks

Journal of

Control Science

and Engineering
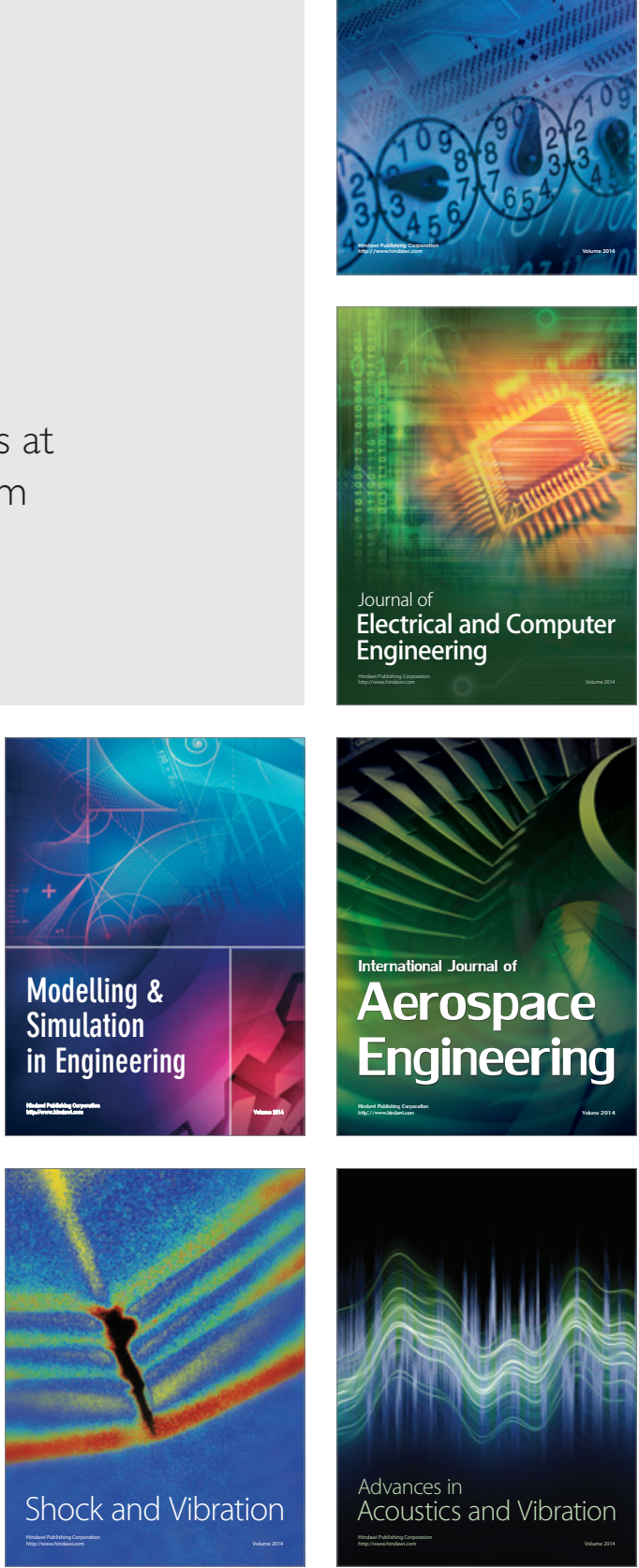